\title{
Selected Open Problems in Graph Drawing
}

\author{
Franz Brandenburg, David Eppstein, Michael T. Goodrich, \\ Stephen Kobourov, Giuseppe Liotta, and Petra Mutzel \\ 1 Univ. of Passau, Germany. \\ brandenburg@informatik.uni-passau.de \\ 2 Univ. of California-Irvine, USA. \\ eppstein@ics.uci.edu \\ 3 Univ. of California-Irvine, USA. \\ goodrich@acm.org \\ 4 Univ. of Arizona, USA. \\ kobourov@cs.arizona.edu \\ ${ }^{5}$ Univ. of Perugia, Italy. \\ liotta@diei.unipg.it \\ ${ }^{6}$ Vienna Univ. of Technology, Austria. \\ mutzel@ads.tuwien.ac.at
}

\begin{abstract}
In this manuscript, we present several challenging and interesting open problems in graph drawing. The goal of the listing in this paper is to stimulate future research in graph drawing.
\end{abstract}

\section{Introduction}

This paper is devoted to exploring some interesting and challenging open problems in graph drawing. We have specifically chosen topics motivated from research themes of current interest in graph drawing with an emphasis on computational geometry, including proximity drawings, 2D and 3D straight-line drawings, 2D and 3D orthogonal representations, crossing minimization, and graph drawing checkers. Our choice of open problems and themes should not be seen as exhaustive, however, as graph drawing is a rich area of research with many open problems and research themes. For example, there are interesting open problems related to the routing of multiple curves in the plane, dynamic graph drawing, drawing of hypergraphs and Venn diagrams, applied graph drawing, and label placement in graph drawings, which we do not specifically address in this paper.

In any survey of open problems, there is naturally going to be a certain emphasis that reflects the perspective of the authors, and this paper is no exception. Nevertheless, we have striven to include open problems motivated by topics we believe are of general interest. That is, we feel that the solution to any of the open problems we include in this paper would be of wide interest in the graph drawing community. 


\section{Proximity Drawability Problems}

Recently, much attention has been devoted to the study of the combinatorial properties of different types of proximity graphs. Proximity graphs, originally defined in the context of computational geometry and pattern recognition, are typically used to describe the shape of a set of points. In the survey by Toussaint [84, such graphs are classified by using the notion of proximity between points: In a proximity graph, two points are connected by an edge if and only if they are deemed close by some proximity measure. Examples of proximity graphs include well-known geometric graphs such as minimum spanning trees, Gabriel graphs, minimum weight triangulations, rectangle of influence graphs, visibility graphs, and Delaunay diagrams [116].

Proximity graphs can be regarded as straight-line drawings that satisfy some additional geometric constraints. Thus the problem of analyzing the combinatorial properties of a given type of proximity graph naturally raises the question of the characterization of those graphs admitting the given type of drawing. This, in turn, leads to the investigation of the design of efficient algorithms for computing such a drawing when one exists. These questions are far from being resolved in general, and only partial answers have appeared in the literature [46, $48,84,95,97,102,103,105$.

\subsection{Realizability of Minimum Spanning Trees in 3D Space}

Among the most challenging questions in this family of graph drawing problems it has to be mentioned one related with drawing trees as minimum spanning trees in 3D. A minimum spanning tree of a set $P$ of points is a connected, straight-line drawing that has $P$ as vertex set and minimizes the total edge length. A tree $T$ can be drawn as a minimum spanning tree if there exists a set $P$ of points such that the minimum spanning tree of $P$ is isomorphic to $T$.

The problem of testing whether a tree can be drawn as a Euclidean minimum spanning tree in the plane is essentially solved. Monma and Suri [110] proved that each tree with maximum vertex degree at most five can be drawn as a minimum spanning tree of some set of vertices by providing a linear time (real RAM) algorithm. In the same paper it is shown that no tree having at least one vertex with degree greater than six can be drawn as a minimum spanning tree. As for trees having maximum degree equal to six, Eades and Whitesides [57] showed that it is NP-hard 65] to decide whether such trees can be drawn as minimum spanning trees. The 3-dimensional counterpart of the problem is however not yet solved. In [101] it is shown that no trees having at least one vertex with degree greater than twelve can be drawn as a Euclidean minimum spanning tree in 3D while all trees with vertex degree at most nine are drawable.

Problem 1: Let $T$ be a tree with maximum vertex degree at most twelve. Is there a polynomial time algorithm to decide whether $T$ can be drawn as a Euclidean minimum spanning tree in $3 D$-space and, if so, compute such a drawing? 


\subsection{Drawing Minimum Weight Triangulations}

A minimum weight drawing of a planar triangulated graph $G$ is a straight-line drawing $\Gamma$ of $G$ with the additional property that $\Gamma$ is a minimum weight triangulation of the points representing the vertices. If a graph admits a minimum weight drawing it is called minimum weight drawable, else it is called minimum weight forbidden.

Little is known about the problem of constructing a minimum weight drawing of a planar triangulation. Moreover, it is still not known whether computing a minimum weight triangulation of a set of points in the plane is NP-hard. Several papers have been published on this last problem, either providing partial solutions, or giving efficient approximation heuristics. A limited list of references includes the work by Meijer and Rappaport [118, Lingas 100, Keil 89], Dickerson et al. [43, Kirkpatrick [90, Aichholzer et al. 2], Cheng and Xu 22], Dickerson and Montague [4], and Levcopoulos and Krznaric 98199].

In 95. Lenhart and Liotta show that all maximal outerplanar triangulations are minimum weight drawable and a linear time (real RAM) drawing algorithm for computing a minimum weight drawing of these graphs was given. In 97] Lenhart and Liotta examine the endoskeleton - or skeleton, for short-of a triangulation: that is, the subgraph induced by the internal vertices of the triangulation. They construct skeletons that cannot appear in any minimum weight drawable triangulation; skeletons that do appear in minimum weight drawable triangulations; and skeletons that guarantee minimum weight drawability.

Besides the natural (and ambitious) goal of characterizing those planar graphs that are minimum weight drawable, we recall here two open problems whose solution would represent an interesting contribution to the investigation of the combinatorial structure of minimum weight triangulations.

In [97] it is shown that any forest can be the skeleton of a minimum weight drawable triangulation. On the other hand, Wang, Chin, and Yang [133] also focus on the minimum weight drawability of triangulations with acyclic skeletons and show examples of triangulations of this type that do not admit a minimum weight drawing. These two results naturally lead to the following problem.

Problem 2: Characterize the class of triangulations with acyclic skeleton that admit a minimum weight drawing.

Lenhart and Liotta [97] also show that there exist an infinite class of minimum weight drawable triangulations that cannot be realized as Delaunay triangulations (that is, for any triangulation $T$ of the class it does not exist a set $P$ of points such that the Delaunay triangulation of $P$ is isomorphic to $T$ ). It is worth remarking that the study of the geometric differences between the minimumweight and Delaunay triangulations of a given set of points in order to compute good approximations of the former has a long tradition (see, e.g., [90998107]), yet little is known about the combinatorial difference between Delaunay triangulations and minimum-weight triangulations. 
Problem 3: Further investigate the combinatorial relationship between minimum weight and Delaunay drawable triangulations. Are there any Delaunay drawable and minimum weight forbidden triangulations?

\subsection{Gabriel Drawability}

Let $P$ be a set of $n$ distinct points in the plane. The Gabriel graph of $P$ is a geometric graph whose vertices are the points of $P$ such that there exists an edge $(u, v)$ if and only if the disk whose antipodal points are $u$ and $v$ does not contain any points of $P$ except $u$ and $v$ (the disk is assumed to be a closed set). Gabriel graphs have been first studied in the context of pattern recognition and computational morphology, where one is given a set of points on the plane and is asked to display the underlying shape of the set by constructing a graph whose vertices are the points and whose edges are segments connecting pairs of points. For references on this topics see, e.g., the survey by Toussaint 84].

More recently, the problem of computing straight-line drawings that have the additional property of being Gabriel graphs of their vertices has been considered. In 13], the problem of characterizing Gabriel-drawable trees has been addressed and an algorithm to compute Gabriel drawings of trees in the plane is given. Lubiw and Sleumer 105] proved that maximal outerplanar graphs admit both relative neighborhood drawings and Gabriel drawings. This result has been extended in [96] to all biconnected outerplanar graphs. Several problems about Gabriel drawings remain open. Among the most fascinating and challenging, we mention the following.

Problem 4: Characterize the family of Gabriel drawable triangulations, that is, the family of those triangulations that admit a straight-line drawing where the angles of each triangular face are less than $\pi / 2$.

\section{Straight-Line Drawability in 2D and 3D}

A classical area of investigation deals with crossing-free straight-line drawings of planar graphs in two and three dimensions. Given a graph $G$, the vertices in a drawing of $G$ are constrained to be located at integer grid points and the optimization goal is that of computing drawings whose area/volume is small. The area/volume of a drawing $\Gamma$ is measured as the number of grid points contained in a bounding box of $\Gamma$, i.e., the smallest axis-aligned box enclosing $\Gamma$.

\subsection{Compact Straight-Line Drawings in 2D Space}

A rich body of literature has been published on computing straight-line drawings of graphs, such that the vertices are the intersection points of an integer $2 \mathrm{D}$ grid and the overall area of the drawing is kept small. Typically, papers that deal with this subject focus on lower bounds on the area required by straightline drawings of specific classes of graphs and on the design of algorithms that 
possibly match these lower bounds. Schnyder [122,123 and de Fraysseix, Pach, and Pollack 29] independently showed that every $n$-vertex triangulated planar graph has a crossing-free straight-line $O(n) \times O(n)$ grid drawing, and that this is worst case optimal. This seminal contribution was followed by related work by Kant 8687], Chrobak and Kant 25], Schnyder and Trotter [124], Felsner 62, and Chrobak, Goodrich, and Tamassia 23. Results on classes of drawings of trees include the results by Garg, Goodrich and Tamassia 68, by Chan 17, and by Garg and Rusu 70. Summarizing tables and more references can be found in the book by Di Battista, Eades, Tamassia, and Tollis 31.

The following problem is motivated by the observation that trees admit straight-line grid drawings with linear or almost-linear area, while triangulations may require a grid of quadratic size.

Problem 5: Find nontrivial classes of graphs with $n$ vertices richer than trees that admit straight-line planar drawings on an integer $2 D$ grid of size $o\left(n^{2}\right)$.

Some evidence that Problem 5 might be solvable for families of graphs other than trees was given in a recent paper by Biedl [9], where she shows that all outerplanar graphs admit an $O(n \log n)$ area drawing; however, the algorithm presented in [9] does not compute straight-line drawings. Very recently, a paper presented at GD 2003 by Garg and Rusu [71 proves that an outerplanar graph with $n$ vertices and degree $d$ has a straight-line grid drawing in area $O\left(d n^{1.693}\right.$.

Additional problems in this area include the following well-known problem, which has been popularized by Xin He:

Problem 6: Given an n-vertex plane triangular graph $G$, what is the smallest area straight-line planar drawing of $G$ such that vertices are drawn at integer grid points?

This problem was first posed by Rosenstiehl and Tarjan [120]. A well-known upper bound is $(n-1) \times(n-1)$ (many such algorithms achieve this), which was recently improved by Zhang and He [136] to $(n-\Delta-1) \times(n-\Delta-1)$, where $0 \leq \Delta \leq\lfloor(n-1) / 2\rfloor$ is a value derived from the cycle structure of $G$. A known lower bound for this problem is provided by the plane graph consisting of a sequence of $n / 3$ nested triangles connected to each other by six edges. It is not hard to see that this graph requires a drawing area of at least $2 n / 3 \times 2 n / 3[29$, 106. Note, that this is not really a lower bound for planar graphs, only for plane graphs (i.e., the combinatorial embedding and the outer face is fixed). Since the nested triangle graph is 3 -connected, it has only one combinatorial embedding (up to its mirror image). However, it is not clear what happens if one is free to pick the outer face.

Problem 7: What is the smallest area grid drawing for the nested triangles graph, when the outer face is not given?

Many planar graph drawing algorithms incrementally construct a drawing based on inserting vertices according to a particular ordering, such as an stnumbering or canonical ordering of the graph's vertices [87]. Such drawings place 
vertices in a numbering-upward fashion, so that listing the vertices according to the ordering lists the vertices by non-decreasing $y$-coordinates.

Problem 8: Is there a polynomial algorithm for numbering and embedding the vertices of a given planar graph $G$ so as to minimize the area of a numberingupward planar drawing of $G$ (taken over all possible numberings of $G$ )?

\subsection{Extensibility and Universality for Planar Graphs}

Consider the following problem: given a planar graph in which some vertices have already been placed in the plane (i.e., a partial embedding), place the remaining vertices to form a straight line embedding of the graph.

Problem 9: Is there a polynomial time algorithm for extending a partial straight-line embedding to a planar straight-line drawing?

This problem also comes up in mesh generation, where the already-placed vertices can be assumed to form a simple polygon and the graph can be assumed to have all interior faces triangles; do these assumptions simplify the problem?

A related problem is the following.

Problem 10: (Drawing with fixed vertex positions) Suppose you are given as input an unlabeled planar graph with $n$ vertices, and a set of $n$ points in the plane. You wish to assign vertices to points to create a planar straight line drawing. What is the computational complexity of this problem?

Concerning the problem above, Bose 12 conjectured it to be NP-complete and solved some special cases in polynomial time. Kaufmann and Wiese 88, show NP-completeness of a related problem in which some bends are allowed.

In general, a set $S$ of points is called a universal set for a family of a graphs $\mathcal{F}$ if every graph in $\mathcal{F}$ can be drawn crossing-free using the points from $S$ for vertex locations.

Problem 11: Does there exist a small universal point set for planar graphs? That is, given $n$, is there a set of $O(n)$ points in the plane, so that every planar graph of $n$ vertices can be drawn with straight-lines and no crossings on this point set? If not, what is the smallest $f(n)$ such that there exists a set of $f(n)$ points such that every $n$-vertex graph can be drawn using these points?

There are several algorithms that show $f(n) \leq n^{2}$. Chrobak and Karloff 26 ] show that $f(n) \geq 1.098 n$, for sufficiently large $n$. Likewise, the following biuniversal set question is also of interest.

Problem 12: Given two planar graphs each on $n$ vertices, can one always find a set of $n$ points, so that each of the two graphs can be embedded with straightlines and no crossings on this set? 


\subsection{Universal Sets in 3D}

While the problem of computing small-sized crossing-free straight-line drawings in the plane has a long tradition, its 3D counterpart has become the subject of much attention only in recent years. Chrobak, Goodrich, and Tamassia 23] gave an algorithm for constructing 3D convex drawings of triconnected planar graphs with $O(n)$ volume and non-integer coordinates. Cohen, Eades, Lin and Ruskey [27] showed that every graph admits a straight-line crossing-free 3D drawing on an integer grid of $O\left(n^{3}\right)$ volume, and proved that this is asymptotically optimum. Calamoneri and Sterbini 15 showed that all 2-, 3-, and 4-colorable graphs can be drawn in a 3D grid of $O\left(n^{2}\right)$ volume with $O(n)$ aspect ratio and proved a lower bound of $\Omega\left(n^{1.5}\right)$ on the volume of such graphs. For $r$-colorable graphs, Pach, Thiele and Tóth [113] showed a bound of $\theta\left(n^{2}\right)$ on the volume. Garg, Tamassia, and Vocca [74] showed that all 4-colorable graphs (and hence all planar graphs) can be drawn in $O\left(n^{1.5}\right)$ volume and with $O(1)$ aspect ratio but using a grid model where the coordinates of the vertices may not be integer.

In a recent paper, Felsner, Liotta, and Wismath [63] approached the drawing problem with the following point of view: Instead of "squeezing" a drawing onto a small portion of a grid of unbounded dimensions, it is assumed that a grid of specified dimensions (involving a function of $n$ ) is given and we consider what the graphs are whose drawings fit that restricted grid. For example, it is well-known that there are families of graphs that require $\Omega\left(n^{2}\right)$ area to be drawn in the plane, the canonical example being a sequence of $n / 3$ nested triangles (see [29, 24[123]), as mentioned above. Such graphs can be drawn on the surface of a three dimensional triangular prism of linear volume and using integer coordinates. Thus a natural question is whether there exist specific restrictions of the 3D integer grid of linear volume that can support straight-line crossing-free drawings of meaningful families of graphs. The following open problem is mentioned in the paper by Felsner, Liotta, and Wismath 63].

Problem 13: Does there exist a 3D universal grid set of linear volume that supports all planar graphs?

Partial results on Problem 13 are as follows. In 63 it is shown that all outerplanar graphs can be drawn in a restricted integer 3D grid of linear volume, called prism. A prism is a subset of the integer 3D grid consisting of three parallel lines through the points $(0,1,0),(0,0,0)$, and $(0,0,1)$. In the same paper it is also shown that a prism does not support all planar graphs and that even adding a fourth parallel line does not suffice in general. Dujmović, Morin, and Wood [50] present $O\left(n \log ^{2} n\right)$ volume drawings of graphs with bounded tree-width and $O(n)$ volume for graphs with bounded path-width. Wood [135] shows that also graphs with bounded queue number have 3D straight-line grid drawings of $O(n)$ volume. A recent result by Dujmović and Wood [51:56] shows that linear volume can also be achieved for graphs with bounded tree-width; they show 3D straight-line grid drawings of volume $c \times n$ for these graphs, where $c$ is a constant whose value exponentially depends on the tree-width. The value of constant $c$ is reduced in the work by Di Giacomo, Liotta, and Meijer [39], although the 
constant still depends exponentially on the tree-width. For the special case of 2-trees (every series-parallel graph can be augmented to be a 2-tree), Dujmović and Wood [51,56 show $36 \times 37 \times 37\left\lceil\frac{n}{18}\right\rceil$ volume. This bound is reduced to $30 \times 31 \times 31\left\lceil\frac{n}{15}\right\rceil$ by Di Giacomo, Liotta, and Meijer [39]. Different families of graphs with constant queue number are studied by Di Giacomo and Meijer [42] who present algorithms that compute linear volume 3D drawings of for these graphs where the multiplicative constants in the volume bounds significantly improve those obtained by directly applying the results in 135. Di Giacomo [38] and Di Giacomo, Liotta, and Wismath [41] show $4 \times n$ and $32 \times n$ volume for two subclasses of series-parallel graphs. A result related to Problem 13 is the elegant work presented at GD 2003 by Dujmović and Wood [54] who describe different families of graphs, including planar graphs, for which a sub-quadratic (but still super-linear) volume upper bound can be found. A list of very recent papers related to Problem 13 also includes 52 5553.

\section{Orthogonal Representations}

An orthogonal drawing of a graph maps its vertices to points on an integer grid and its edges to a sequence of alternating axis-parallel segments. The study of the orthogonal drawing convention has a very long tradition in the graph drawing literature, because of the several applications of this convention in a variety of fields. For an introduction on orthogonal drawings and their several applications, see 31. It is immediate to see that in order for an orthogonal drawing to exist, the degree of the vertices must not exceed four in the plane and six in three dimensions.

\subsection{Orthogonal Representations in 2D Space}

The topology-shape-metrics approach 31 for constructing a planar orthogonal drawing of a planar graph in $2 D$ consists of three main steps, called planarization, orthogonalization, and compaction. The planarization step determines an embedding, i.e., the face cycles, for the graph in the plane. The orthogonalization step determines an orthogonal representation of the input graph, i.e., a labeling for each edge $(u, v)$ of the graph that defines the shape of $(u, v)$ in the final drawing. For example, $(u, v)$ could be labeled $R L L L R$, which would say "starting from $u$ first turn right, then turn left three times, then right." Finally, the compaction step computes the drawing, giving coordinates to vertices and bends while preserving the shape of the edges determined in the orthogonalization step. For each step of the approach, different optimization problems (for example minimizing the number of bends, minimizing the area, minimizing the maximum edge length) have been studied, and papers providing optimal algorithms and effective heuristics have been presented (see, e.g., [6 1119291]).

A bend-minimum planar orthogonal drawing of a plane graph $G$ is one which has the minimum number of bends along the edges among all possible planar orthogonal drawings of $G$. The problem of computing bend-minimum planar 
orthogonal drawings is among the most famous in the graph drawing literature and has been studied both in the fixed embedding setting and in the variable embedding setting.

In the fixed embedding setting, the input is a planar graph $G$ together with a planar embedding (i.e., a circular ordering of the edges incident on each vertex of $G$ and an outer face); the output is a planar orthogonal drawing of $G$ that has the minimum number of bends among all drawings which maintain the given embedding of $G$. A well-known seminal paper by Tamassia 128. shows that such a drawing of $G$ can be computed in $O\left(n^{2} \log n\right)$ time by mapping the problem to computing a flow of minimum cost on a suitable network. The time complexity bound is further improved by Garg and Tamassia 72] who present an $O\left(n^{1.75} \log n\right)$ time algorithm.

Problem 14: Let $G$ be a 4-planar graph (i.e., a planar graph whose maximum vertex degree is at most four) with a given planar embedding $\Phi$. Is there an algorithm that computes a bend-minimum orthogonal representation of $G$ preserving $\Phi$ and has time complexity o $\left(n^{1.75} \log n\right) ?$

Notice that the choice of a planar embedding of $G$ can deeply affect the resulting number of bends in the orthogonal representation. For example, the algorithm in 128. can give rise to orthogonal representations of the same graph that, depending on the choice of its planar embedding, differ in the number of bends by even a linear factor [36]. Thus, there is natural interest in algorithms that work in a variable embedding setting, i.e., algorithms that are allowed to change the planar embedding of the input graph in order to minimize the bends.

The variable embedding version of the bend-minimization problem is unfortunately harder than the fixed embedding one. Namely, Garg and Tamassia [73] have proved that computing a bend-minimum planar orthogonal drawing in the variable embedding setting is NP-hard. Di Battista, Liotta, and Vargiu [36] proved that the problem can be solved in $O\left(n^{3}\right)$ time if the input graph is a series-parallel graph and that it can be solved in $O\left(n^{5} \log n\right)$ time if the input is a 3-planar graph (i.e., a graph that has vertex degree at most three). Garg and Liotta [69] further reduced this time complexity, but at the expenses of a few extra bends in the computed representation. Namely, they present an $O\left(n^{2}\right)$-time algorithm that receives as input a 3-planar graph $G$ with $n$ vertices and computes an orthogonal representation of $G$ with at most 3 bends more than the minimum number of bends.

The natural counterpart of Problem 14 in the variable embedding setting is therefore the following.

Problem 15: Let $G$ be a planar graph whose vertices have degree at most three. Is there an algorithm to compute a planar bend-minimum orthogonal drawing of $G$ in $o\left(n^{5} \log n\right)$ time?

Very recently, a paper presented at GD 2003 by Rahman, Egi, and Nishizeki [117] partially answers Problem[15] by showing a linear-time algorithm for subdivisions of planar triconnected cubic graphs. 
Because of the NP-hardness of finding the best planar embedding concerning the number of bends, recent papers have focussed on identifying good embeddings. Pizzonia and Tamassia [115] have suggested to consider planar embeddings with the minimum depth. The depth of an embedding measures the topological nesting of the biconnected components of $G$ in the embedding. They have also given a polynomial time algorithm for computing the minimum depth embedding in the case when the embeddings of the biconnected components are fixed. Recently, Gutwenger and Mutzel [80] have suggested a linear time algorithm which computes the minimum depth embedding over the class of all planar embeddings without any restriction. They also suggest to look for embeddings with maximum outer face, and provide a linear time algorithm. Moreover, they give a linear time algorithm for the combined problem, namely, to search the embedding with maximum outer face among the set of all minimum depth embeddings. However, so far, no experiments or proofs exist that show that embeddings satisfying these criteria (almost) always lead to good drawings. The research in this direction has just started.

Problem 16: Identify (provable) criteria for planar embeddings that lead to good drawings, e.g., that lead to drawings with a relatively small number of bends and/or small area. Moreover, come up with corresponding polynomial time algorithms.

\subsection{Compact Orthogonal Layouts}

As in other drawing problems, there is considerable interest in the exact area bounds for various orthogonal drawing problems.

Problem 17: What are exact bounds for the area of binary tree layouts in each of the following scenarios:

1. straight-line drawings on the grid

2. rectangular drawings on the grid (H-layouts)

3. straight-line upward drawings on the grid

4. rectangular upward drawings on the grid (T-layouts)

Problem 18: Does every binary tree have a drawing with straight-line edges in $O(n)$ area such that all nodes are placed on grid points?

In the rectangular case, the straight-line edges are either horizontal or vertical. In the upward case, the edges are $y$-monotone or go in directions west, east, and north (south is excluded). Known results are $O(n \log \log n)$ area for straight-line drawings, $O(n \log \log n)$ for straight-line upward drawings [68 125], $O(n \log \log n)$ for rectangular drawings [18, and $O(n \log n)$ for upward rectangular (from hv drawings). 
Problem 19: What are exact bounds for the area of upward (strictly upward) layouts of binary trees. Specifically, what is the complexity of determining, given a binary tree $T$ and a bound $K$, whether there exists a rectangular upward (strictly upward, hv) layout with area at most $K$ ? Are these problems NP-hard?

Known results: NP-hardness is known for "H"-tree layouts (all 4 directions) [7] and improved to binary trees by Gregori [76], for upward drawings of ternary trees by Edler [58], and for rectilinear layouts by Garg and Tamassia [73].

\subsection{Orthogonal Representations in 3D Space}

An essential prerequisite of the topology-shape-metrics approach to drawing graphs in $2 \mathrm{D}$ is a characterization of $2 \mathrm{D}$ orthogonal representation, i.e., a characterization of those graphs with edges labeled by orthogonal directions that can be drawn without crossings, while respecting the desired shapes for the edges. This problem has been studied in several papers, including [128|130, and has also been generalized to non-orthogonal polygons and graphs in [131/37 67. However, while the literature on 3D orthogonal drawings is quite rich, the extension of the topology-shape-metrics approach to 3D has so far remained an elusive target. A major difficulty is that in $3 \mathrm{D}$, there is no counterpart to the $2 \mathrm{D}$ characterization of orthogonal representations.

A direction label is a label in the set $\{E, W, N, S, U, D\}$, where each label specifies a direction East, West, North, South, Up, or Down, respectively. Let $G$ be a graph such that each edge $(u, v)$ of $G$ is associated with two opposite orientations called darts of $(u, v)$. A $3 D$ shape graph is a labeling $\sigma$ of the darts of $G$ such that:

- Each dart is associated with exactly one direction label.

- For each edge $e$ of $G$ the two opposite darts of $e$ have labels which specify opposite directions.

- Each vertex does not have two entering darts with the same label.

Shape graph $\gamma$ is said to be a $3 D$ orthogonal representation if there exists an orthogonal drawing $\Gamma$ of $G$ so that $\Gamma$ is simple (i.e., no two edges of $\Gamma$ share any points except common endpoints) and satisfies the direction constraints on its edges as specified by $\sigma$.

Problem 20: Characterize those shape graphs that are 3D orthogonal representations.

Only very preliminary results toward finding such characterization have so far been discovered. Di Battista, Liotta, Lubiw, and Whitesides [35] solve the problem for paths (with the additional constraint that one endpoint must be drawn at the origin and the other in a given octant) and for shape cycles [34]. As for structurally more complex graphs, Di Giacomo, Liotta, and Patrignani [40] show that the characterization for shape cycles does not extend to general 
graphs, even for apparently simple structures such as theta graphs (a theta graph is a simple graph consisting of three cycles). In the same paper, the authors present a sufficient condition under which a shape theta graph is a $3 \mathrm{D}$ orthogonal representation. A consequence of the work in 40 is the following question, whose answer may be an important intermediate step toward the ambitious goal of solving Problem 20.

Problem 21: Characterize those shape theta graphs that are 3D orthogonal representations.

\section{Crossing Minimization}

The crossing number $\operatorname{cr}(G)$ of a graph $G$ is the minimum number of edge crossings in any drawing of $G$. It represents a fundamental measure of nonplanarity of graphs and has been studied for more than 40 years. Nevertheless, so far only few infinite classes of graphs exist for which the crossing number is known. We do not even know if the long open standing conjectures for the complete graph $K_{n}$ and for the complete bipartite graph $K_{m, n}$ are true. So far, the correctness has been verified only for $K_{n}$ with $n \leq 10$ and $K_{m, n}$ with $m \leq 6$ as well as a few more special cases (see, e.g., [119]).

Problem 22: Determine the crossing number for the complete graph $K_{n}$ and for the complete bipartite graph $K_{m, n}$.

The crossing number problem and many of its variants are NP-hard. So far, no algorithms exist which are able to compute the crossing number of even small graphs in practice (like, e.g., branch-and-bound algorithms). The main problem with exact, enumeration-based, methods is the lack of tight lower bounds. Most lower bounds only depend on the number of vertices and edges of the graph, but not on its particular structural properties. Research results in the latter direction have been provided by Leighton 93, Pach, Shahrokhi, and Szegedy 112, Sýkora and Vrťo [127], and Dijdjev and Vrťo [45] who have shown bounds depending on the bisection width and the cut width, respectively. Both problems are NP-hard, too, but they are easier to deal with.

Problem 23: Find tight lower bounds for the crossing number based on structural properties of graphs.

So far, not even efficient approximation algorithms have been found. For graphs with bounded degrees, Even, Guha, and Schieber [60] have suggested an approximation algorithm in which the sum of the numbers of vertices and crossings is $O\left(\log ^{3}|V|\right)$ times the minimum sum thus improving the results of $O\left(\log ^{4}|V|\right)$ by Bhatt and Leighton [8] and Leighton and Rao [94]. A promising approach could be to search for efficient fixed-parameter algorithms. Grohe [77] has given an exact quadratic time algorithm if the number of crossings is consider to be a fixed (small) parameter. Unfortunately, the constant in the worst case time analysis is too big to be useful in practice. 
Problem 24: Provide approximation algorithms or efficient fixed-parameter algorithms for the crossing number problem.

Since the crossing number is so hard to address, researchers started to study variants of the crossing number that might be easier to attack. Pach and Toth [114 have introduced the pairwise crossing number $\operatorname{cr}_{p}(G)$, which is the minimum number of pairs of edges that cross at least once, and the odd crossing number $\mathrm{cr}_{o}(G)$, which is the minimum number of pairs of edges that cross an odd number of times. They have shown that

$$
c r_{o}(G) \leq c r_{p}(G) \leq c r(G) \leq 2\left(c r_{o}(G)\right)^{2}
$$

So far, it is not clear if equality holds.

Problem 25: Prove or disprove: $\operatorname{cr}_{o}(G)=\operatorname{cr}_{p}(G)=\operatorname{cr}(G)$.

Better known is the rectilinear crossing number, which is the minimum number of crossings in a straight-line drawing. It has been shown in Bienstock and Dean 10] that there are graphs with crossing number 4, whose rectilinear crossing numbers are arbitrarily large. Although, it seems easier to study the rectilinear crossing number, also here, the value of the complete graph $K_{n}$ is not known for $n>12$ [.

Problem 26: What is the rectilinear crossing number of $K_{n}$ ?

Closely related to the rectilinear crossing number of complete graphs are Sylvester's Four Point Problem and the problem of finding the minimum number of convex quadrilaterals determined by $n$ points in general position in the plane 104121 .

Aronov et al. 44 showed an $\Omega(\sqrt{n})$ bound for the size of crossing families (i.e., each edge crosses each other), and asked whether a linear sized crossing family always exists.

Problem 27: In a straight-line drawing of the complete graph $K_{n}$, how large must a set of mutually crossing edges be?

A successful approach for computing a small crossing number in practice is the planarization approach (see, e.g., 3279]). Here, in a first step, the minimum number of edges is deleted from $G$ in order to obtain a planar graph $P$. In a second step, the edges are reinserted into $P$ while trying to keep the number of crossings small.

Finding the largest cardinality planar subgraph of a graph is NP-hard, and even MAXSNP-hard, so one cannot expect to approximate it arbitrarily well, but there may be room for reducing the best known approximation ratio of 2.25, which has been achieved by an algorithm by Calinescu et al. [16].

Problem 28: Find an efficient approximation algorithm for the maximum cardinality planar subgraph problem with factor below 2.25. 
Also the edge reinsertion step is a NP-hard optimization problem. The standard algorithm reinserts the edges iteratively into a fixed combinatorial embeding of the planar subgraph $P$. This can be done easily via simple shortest-path computations in the extended dual graph. However, the quality of the resulting drawing highly depends on the chosen embedding for $P$. Gutwenger, Mutzel and Weiskircher 81 have suggested a linear time algorithm for inserting one edge into a planar graph $P$ so that the number of crossings in $P \cup\{e\}$ over the set of all possible planar embeddings of $P$ is minimized. A generalization of this approach for fixed $k$ would be of interest theoretically and practically.

Problem 29: Find a polynomial time algorithm for inserting a fixed number of edges $e_{1}, \ldots, e_{k}$ into a planar graph such that the number of crossings in $P \cup\left\{e_{1}, \ldots, e_{k}\right\}$ over the set of all possible planar embeddings of $P$ is minimized.

\section{Graph Drawing Checkers}

The intrinsic structural complexity of the implementation of geometric algorithms makes the problem of formally proving the correctness of the code unfeasible in most of the cases. This has been motivating the research on checkers. A checker is an algorithm that receives as input a geometric structure and a predicate stating a property that should hold for the structure. The task of the checker is to verify whether the structure satisfies or not the given property. Here, the expectation is that it is often easier to evaluate the quality of the output than the correctness of the software that produces it. Different papers (see, e.g., [30,109]) have agreed on the basic features that a "good" checker should have:

Correctness: The checker should be correct beyond any reasonable doubt. Otherwise, one would incur in the problem of checking the checker.

Simplicity: The implementation should be straightforward.

Efficiency: The expectation is to have a checker that is more efficient than the algorithm that produces the geometric structure.

Robustness: The checker should be able to handle degenerate configurations of the input and should not be affected by errors in the flow of control due to round-off approximations.

Checking is especially relevant in the graph drawing context. Indeed, graph drawing algorithms are among the most sophisticated of the entire computational geometry field, and their goal is to construct complex geometric structures with specific properties. Also, because of their immediate impact on application areas, graph drawing algorithms are usually implemented right after they have been devised. Of course, the checking problem becomes crucial when the drawing algorithm deals with very large data sets, when a simple complete visual inspection of the drawing is difficult or unfeasible.

Devising graph drawing checkers involves answering only apparently innocent questions like: "is this drawing planar?" or "is this drawing upward?" or "are the faces convex polygons?". 
The problem of checking the planarity of a subdivision has been independently studied by Mehlhorn et al. 109] and by Devillers et al. [30. In these papers linear time algorithms are given to check the planarity of a subdivision composed by convex faces. The inputs are the subdivision plus its topological embedding in terms of the ordered adjacency lists of the edges. Unfortunately, extending the above techniques to checking the planarity of a subdivision whose faces are not constrained to be convex, relies on the usage of algorithms for testing the simplicity of a polygon. The only general linear time algorithm known for this problem is the well-known and fairly complex algorithm by Chazelle [20]. Hence, devising a checker based on such algorithm would not satisfy the simplicity requirement. The algorithm in 20] tests the simplicity of a polygon by means of an intermediate triangulation step. Alternative algorithms that can triangulate in linear time special classes of polygons have been devised. See e.g. [5966, 134]. Other almost optimal algorithms can be found in [21:83129].

Problem 30: Let $\Gamma$ be a straight-line drawing of a connected graph $G$ with its combinatorial embedding (i.e., the circular ordering of the edges incident on each vertex of $\Gamma$ ). Devise a simple, robust, and efficient checker for testing the planarity of $\Gamma$.

An example of checker of the type described by Problem 30 can be found in a paper by Di Battista and Liotta 33. who check the upward planarity of straight-line oriented drawings whose faces may not be convex polygons. They introduce regular upward planar embeddings and show that such embeddings coincide with those having a "unique" including planar st-digraph. The concept of regularity is exploited to investigate the relationships between topology and geometry of upward planar drawings. In particular, it is shown that an upward drawing of a regular planar upward embedding satisfies strong constraints on the left-to-right ordering of the edges. Based upon the above results and under the assumption of regularity, a simple and robust linear time checker is presented that tests whether a given drawing $\Gamma$ is upward planar without using any polygon triangulation routine.

Besides planarity, several other checking problems remain open in graph drawing. Indeed, all graph drawing algorithms guarantee certain geometric properties for the drawings they produce. Such properties are usually called "graphic standards" or "drawing conventions". Some of them appear to be easy to check, while others like checking proximity drawings seem to be more challenging. For example, consider the following problem.

Problem 31: Let $\Gamma$ be a straight-line drawing of a tree (or even a simple chain). Is there a robust and simple algorithm to check in $o(n \log n)$ time whether $\Gamma$ is a Euclidean minimum spanning tree of the set of its vertices?

\section{Visualizing Graph Properties}

In this section we consider open problems that are related to the visualization of various graph properties or additional information associated with a graph. 


\subsection{Layered Graphs}

Some graphs are labeled so as to partition the set of vertices into layers. Drawing algorithms that deal with this additional information usually seek to assign the vertices of each layer to a shared $y$-coordinate that is greater than that of the previous layer. Algorithms for drawing layered graphs usually are based on an approach that is commonly referred to as the Sugiyama algorithm [126].

A crucial problem is the layered crossing minimization problem, in which for each layer $L$ permutations of the vertices in $L$ are searched with the goal of obtaining the minimum nmber of crossings when the layers are parallel horizontal lines, all vertices are drawn on the line corresponding to their layers with $x$ coordinate compatible with their position in the layer permutation and all edges are drawn as straight line segments between consecutive layers. The layered crossing minimization problem is NP-hard, even when restricted to two layers. Exact approaches have been suggested by Healy and Kuusik [82] and Jünger et al. 85], but so far they are not yet efficient enough to be used in practical graph layout systems. So far, no polynomial time approximation algorithms exist. Relaxing the layering condition and only requiring that all the directed edges point downwards, then the algorithm by Dujmovic et al. 49] can be used to determine whether a given graph on $n$ vertices can be drawn on $h$ layers with at most $k$ crossings, where $h$ and $k$ are fixed parameters. However, due to the high constants, this algorithm is rather of theoretical nature.

Problem 32: Design a practically efficient approximation algorithm (or fixedparameter algorithm or exact algorithm) for layered crossing minimization.

In practice, the layered crossing minimization problem is solved via a series of two-layer problems. In this context, the task of efficiently counting the crossings in a bilayered graph $G=(V, E)$ is essential. The best known algorithm for counting the crossings for a set of straight line segments in the plane is due to Chazelle [19] and runs in time $O\left(|E|^{1.695}\right)$. For the bilayer cross counting problem, a popular alternative in graph drawing is a sweep-line algorithm running in time $O(|C|+|E|)$, where $|C|$ denotes the number of crossings. A breakthrough has been achieved by Waddle and Malhotra [132 who have provided the first algorithm running in time $O(|E| \log |V|)$. The idea is based on a sweep-line procedure and involves complicated case distinctions. Recently, Barth, Jünger and Mutzel [5] have observed that the bilayer cross counting problem can be reduced to counting the inversions of a certain sequence, which directly lead to a set of simple $O(|E| \log |V|)$ algorithms (e.g., a variant of merge sort). Using the accumulator tree, they can improve the running time to $O\left(|E| \log \left|V_{s}\right|\right)$, where $V_{s}$ is the smaller set of the two layers. In this context, the following problems arise:

Problem 33: Is it possible to count the number of crossings in a bilayered graph faster than in $\Theta(|E| \log |V|)$ time? Is it possible to count the inversions in a sequence of length $n$ faster than $\Theta(n \log n)$ ?

Another issue regarding layered drawings is the representational complexity of describing a layered drawing. 
Problem 34: If a multi-layer drawing of a graph is made in which all the vertices have integer coordinates, how big do those integers need to be? (For planar graphs, it is known that all coordinates can be $O(n)$.)

\subsection{Clustered Graph Drawing}

A clustered graph $C=(G, T)$ consists of an undirected graph $G$ and a rooted tree $T$ in which the leaves of $T$ correspond to the vertices of $G=(V, E)$. Each vertex $c$ in $T$ corresponds to a subset of vertices of the graph called a cluster. $c$ planarity is a natural extension of graph planarity for clustered graphs, and plays an important role in automatic graph drawing. However, the complexity status of c-planarity testing is unknown. Feng, Cohen, and Eades 64] and Dahlhaus [28] have shown that c-planarity can be tested in polynomial time for graphs in which all the cluster induced subgraphs are connected. Recently, Gutwenger et al. [78] have further extended the class of graphs.

Problem 35: Can c-planarity for clustered graphs be tested in polynomial time?

\subsection{Geometric Thickness}

The geometric thickness of a graph is the minimum number of subgraphs into which the graph can be partitioned, in such a way that all subgraphs have planar straight line drawings with the same vertex positions in each drawing. Useful references for the problems that follow are [47108].

Problem 36: The complete graphs $K_{n}$ should require a number of layers of the form $c n$, for some constant $c$. What is $c$ ? Similarly, what is the asymptotic number of layers needed for $K_{n, n}$ ?

For arbitrary graphs, computing the thickness is known to be NP-complete.

Problem 37: Is it equally hard to compute the geometric thickness? Can geometric thickness be approximated efficiently?

Problem 38: Which minor-closed graph families have bounded geometric thickness? Do bounded-degree graphs have bounded geometric thickness?

The solution of the first part of Problem 38 has been announced by Blankenship and Oporowski [11]. Using results of Heath and a structure theorem of Robertson and Seymour, they have shown an even stronger result, namely, that for every minor-closed class of graphs, other than the class of all graphs, there is an integer $K$ such that all members of the class have book thickness at most $K$. 


\subsection{Alternative Graph Representations}

There are many additional research themes in graph drawing that consider alternative representations than the one that assigns vertices to points and edges to curves joining those points. The following problems fall into the category of visibility graph recognition problems.

Problem 39: Is there a polynomial algorithm that takes as input a graph and outputs a representation in the form of a closed simple polygon, with the graph's vertices placed at polygon corners, such that an edge is present in the graph if and only if the line segment between the vertices is contained in the polygon?

An interesting class of problems looks for small representations of dense graphs. For example, given a simple polygon $P$, the visibility graph $G$ for $P$ is the graph having vertex set the same as $P$ and such that there is an edge $(v, w)$ in $G$ if and only if the line segment joining $v$ and $w$ never crosses the boundary of $P$. The following problem 1 is classic [161/75].

Problem 40: Is there a polynomial algorithm for determining, given a graph $G$ and Hamiltonian cycle $C$ in $G$, if there exists a polygon $P$ with $C$ as its boundary and having $G$ as its visibility graph?

The following problem falls into the class of incidence graph recognition problems.

Problem 41: Which graphs can be represented as incidence graphs of line segments in the plane? Does every planar graph have such a representation?

This problem is related to Conway's famous thrackle conjecture. A thrackle is a planar drawing of a graph of $n$ vertices by edges which are smooth curves between the vertices, with the condition that any two edges intersect at exactly one point (including adjacent edges). Conway conjectured that the number of edges in a thrackle cannot exceed $n$. The best proven upper bound is $1.5(n-1)$ (see [14]).

Problem 42: Prove or disprove Conway's thrackle conjecture.

Acknowledgements. We wish to thank Emilio Di Giacomo for useful discussions and comments while preparing this collection of open problems. We also want to thank the anonymous reviewers for their valuable comments and suggestions.

\footnotetext{
${ }^{1}$ http://cs.smith.edu/ orourke/TOPP/P30.html\#Problem.17

${ }^{2}$ http://www.cs.unt.edu/ cbms/CBMS
} 


\section{References}

1. J. Abello and K. Kumar. Visibility graphs and oriented matroids. In R. Tamassia and I. G. Tollis, editors, Graph Drawing (Proc. GD '94), volume 894 of Lecture Notes Comput. Sci., pages 147-158. Springer-Verlag, 1995.

2. O. Aichholzer, F. Aurenhammer, S.-W. Chen, N. Katoh, M. Taschwer, G. Rote, and Y.-F. Xu. Triangulations intersect nicely. Discrete Comput. Geom., 16:339359, 1996.

3. O. Aichholzer, F. Aurenhammer, and H. Krasser. On the crossing number of complete graphs. In Proceedings of the 18th ACM Symposium on Computational Geometry, pages 19-24. ACM Press, 2002.

4. B. Aronov, P. Erdős, W. Goddard, D. J. Kleitman, M. Klugerman, J. Pach, and L. J. Schulman. Crossing families. Combinatorica, 14:127-134, 1994.

5. W. Barth, M. Jünger, and P. Mutzel. Simple and efficient cross counting. In M. Goodrich and S. Kobourov, editors, Graph Drawing (Proc. GD'02), volume 2528 of Lecture Notes Comput. Sci., pages 130-141. Springer-Verlag, 2002.

6. P. Bertolazzi, G. Di Battista, and W. Didimo. Computing orthogonal drawings with the minimum number of bends. In WADS '97, volume 1272 of Lecture Notes in Comput. Sci., pages 331-344, 1998.

7. S. Bhatt and S. Cosmadakis. The complexity of minimizing wire lengths in VLSI layouts. Inform. Process. Lett., 25:263-267, 1987.

8. S. N. Bhatt and F. T. Leighton. A framework for solving VLSI graph layout problems. J. Comput. Syst. Sci., 28:300-343, 1984.

9. T. Biedl. Drawing outer-planar graphs in $O(n \log n)$ area. In M. Goodrich, editor, Graph Drawing (Proc. GD'02), volume 2528 of Lecture Notes Comput. Sci, pages 54-65. Springer-Verlag, 2002.

10. D. Bienstock and N. Dean. Bounds for rectilinear crossing numbers. J. Graph Theory, 17:333, 1993.

11. R. Blankenship and B. Oporowski. Book embeddings of graphs and minor-closed classes. In Thirty-Second Southeastern Internat. Conf. Combinatorics, Graph Theory and Computing, page 30, Baton Rouge, Dept. of Mathematics, Louisiana State University, 2001. http://www.math.lsu.edu/ conf_se/program.pdf.

12. P. Bose. On embedding an outer-planar graph in a point set. Comput. Geom., 23(3):303-312, 2002.

13. P. Bose, W. Lenhart, and G. Liotta. Characterizing proximity trees. Algorithmica, 16:83-110, 1996.

14. G. Cairns and Y. Nikolayevsky. Bounds for generalized thrackles. Discrete Comput. Geom., 23(2):191-206, 2000.

15. T. Calamoneri and A. Sterbini. 3D straight-line grid drawing of 4-colorable graphs. Inform. Process. Lett., 63(2):97-102, 1997.

16. G. Calinescu, C. G. Fernandes, U. Finkler, and H. Karloff. A better approximation algorithm for finding planar subgraphs. In Proc. 7th ACM-SIAM Symp. on Discrete Algorithms (SODA), pages 16-25, 1996.

17. T. Chan. A near-linear area bound for drawing binary trees. Algorithmica, 34:113, 2001.

18. T. M. Chan, M. T. Goodrich, S. R. Kosaraju, and R. Tamassia. Optimizing area and aspect ratio in straight-line orthogonal tree drawings. In S. North, editor, Graph Drawing (Proc. GD '96), volume 1190 of Lecture Notes Comput. Sci., pages 63-75. Springer-Verlag, 1997. 
19. B. Chazelle. Reporting and counting segment intersections. J. Comput. Syst. Sci., 32:156-182, 1986.

20. B. Chazelle. Triangulating a simple polygon in linear time. Discrete Comput. Geom., 6:485-524, 1991.

21. B. Chazelle and J. Incerpi. Triangulation and shape-complexity. ACM Trans. Graph., 3(2):135-152, 1984.

22. S.-W. Cheng and Y.-F. Xu. On $\beta$-skeleton as a subgraph of the minimum weight triangulation. Theoretical Computer Science, 262(1-2):459-471, 2001.

23. M. Chrobak, M. T. Goodrich, and R. Tamassia. Convex drawings of graphs in two and three dimensions. In Proc. 12th Annu. ACM Sympos. Comput. Geom., pages 319-328, 1996.

24. M. Chrobak and S. ichi Nakano. Minimum-width grid drawings of plane graphs. Comput. Geom. Theory Appl., 11:29-54, 1998.

25. M. Chrobak and G. Kant. Convex grid drawings of 3-connected planar graphs. Internat. J. Comput. Geom. Appl., 7(3):211-223, 1997.

26. M. Chrobak and H. Karloff. A lower bound on the size of universal sets for planar graphs. SIGACT News, 20, 1989.

27. R. F. Cohen, P. Eades, T. Lin, and F. Ruskey. Three-dimensional graph drawing. Algorithmica, 17:199-208, 1997.

28. E. Dahlhaus. Linear time algorithm to recognize clustered planar graphs and its parallelization. In C. Lucchesi, editor, 3rd Latin American Symposium on Theoretical Informatics (LATIN '98), volume 1380 of Lecture Notes Comput. Sci., pages 239-248. Springer-Verlag, 1998.

29. H. de Fraysseix, J. Pach, and R. Pollack. How to draw a planar graph on a grid. Combinatorica, 10(1):41-51, 1990.

30. O. Devillers, G. Liotta, F. P. Preparata, and R. Tamassia. Checking the convexity of polytopes and the planarity of subdivisions. Comput. Geom. Theory Appl., 11:187-208, 1998.

31. G. Di Battista, P. Eades, R. Tamassia, and I. G. Tollis. Graph Drawing. Prentice Hall, Upper Saddle River, NJ, 1999.

32. G. Di Battista, A. Garg, G. Liotta, R. Tamassia, E. Tassinari, and F. Vargiu. An experimental comparison of four graph drawing algorithms. Comput. Geom. Theory Appl., 7:303-325, 1997.

33. G. Di Battista and G. Liotta. Upward planarity checking: "Faces are more than polygons". In S. Whitesides, editor, Graph Drawing (Proc. GD '98), Lecture Notes Comput. Sci., pages 72-86. Springer-Verlag, 1998.

34. G. Di Battista, G. Liotta, A. Lubiw, and S. Whitesides. Orthogonal drawings of cycles in 3D space. In J. Marks, editor, Graph Drawing (Proc. GD '00), Lecture Notes Comput. Sci. Springer-Verlag, 2000.

35. G. Di Battista, G. Liotta, A. Lubiw, and S. Whitesides. Embedding problems for paths with direction constrained edges. Theoretical Computer Science, 289(2):897-917, 2002.

36. G. Di Battista, G. Liotta, and F. Vargiu. Spirality and optimal orhogonal drawings. SIAM J. Comput., 27(6):1764-1811, 1998.

37. G. Di Battista and L. Vismara. Angles of planar triangular graphs. SIAM J. Discrete Math., 9(3):349-359, 1996.

38. E. Di Giacomo. Drawing series-parallel graphs on restricted integer 3D grids. In Graph Drawing (Proc. GD 03), Lecture Notes in Comput. Sci., 2003. These proceedings.

39. E. Di Giacomo, G. Liotta, and H. Meijer. 3D straight-line drawings of $k$-trees. Technical Report TR-2003-473, Queen's University, School of Computing, 2003. 
40. E. Di Giacomo, G. Liotta, and M. Patrignani. Orthogonal 3D shapes of theta graphs. In M. Goodrich, editor, Graph Drawing (Proc. GD'02), volume 2528 of Lecture Notes Comput. Sci., pages 142-149. Springer-Verlag, 2002.

41. E. Di Giacomo, G. Liotta, and S. Wismath. Drawing series-parallel graphs on a box. In 14th Canadian Conference On Computational Geometry (CCCG '02), 2002.

42. E. Di Giacomo and H. Meijer. Track drawings of graphs with constant queue number. These proceedings, 2003.

43. M. Dickerson, J. Keil, and M. Montague. A large subgraph of the minimum weight triangulation. Discrete and Computational Geometry, 18:289-304, 1997.

44. M. T. Dickerson and M. H. Montague. A (usually?) connected subgraph of the minimum weight triangulation. In Proc. 12th Annu. ACM Sympos. Comput. Geom., pages 204-213, 1996.

45. H. Dijdjev and I. Vrťo. An improved lower bound for crossing numbers. In M. Jünger, S. Leipert, and P. Mutzel, editors, Graph Drawing (Proc. GD '01), Lecture Notes in Comput. Sci. Springer-Verlag, 2001. to appear.

46. M. B. Dillencourt. Realizability of Delaunay triangulations. Inform. Process. Lett., 33(6):283-287, Feb. 1990.

47. M. B. Dillencourt, D. Eppstein, and D. S. Hirschberg. Geometric thickness of complete graphs. J. Graph Algorithms \& Applications, 4(3):5-17, 2000.

48. M. B. Dillencourt and W. D. Smith. Graph-theoretical conditions for inscribability and Delaunay realizability. In Proc. 6th Canad. Conf. Comput. Geom., pages 287-292, 1994.

49. V. Dujmović, M. Fellows, M. Hallett, M. Kitching, G. Liotta, C. McCartin, N. Nishimura, P. Radge, F. Rosamond, M. Suderman, S. Whitesides, and D. Wood. On the parameterized complexity of layered graph drawing. In F. M. auf der Heide, editor, Algorithms, 9th European Symposium (ESA '01), volume 2161 of Lecture Notes in Comput. Sci., pages 488-499. Springer-Verlag, 2001.

50. V. Dujmović, P. Morin, and D. Wood. Pathwidth and three-dimensional straight line grid drawings of graphs. In M. T. Goodrich and S. Koburov, editors, Graph Drawing (Proc. GD '02), volume 2528 of Lecture Notes Comput. Sci., pages 42-53. Springer-Verlag, 2002.

51. V. Dujmović and D. R. Wood. Tree-partitions of $k$-trees with applications in graph layout. Technical Report 02-03, Dept. Comput. Sci., Carleton Univ., Ottawa, Canada, 2002.

52. V. Dujmović and D. R. Wood. On linear layouts of graphs. Technical Report 03-05, Dept. Comput. Sci., Carleton Univ., Ottawa, Canada, 2003.

53. V. Dujmović and D. R. Wood. Stacks, queues and tracks: Layouts of graphs subdivisions. Technical Report 03-07, Dept. Comput. Sci., Carleton Univ., Ottawa, Canada, 2003.

54. V. Dujmović and D. R. Wood. Three-dimensional grid drawings with subquadratic volume. In Graph Drawing (Proc. GD 03), Lecture Notes in Comput. Sci., 2003. These proceedings.

55. V. Dujmović and D. R. Wood. Track layouts of graphs. Technical Report 03-06, Dept. Comput. Sci., Carleton Univ., Ottawa, Canada, 2003.

56. V. Dujmović and D. R. Wood. Tree-partitions of $k$-trees with application in graph layout. In Workshop on Graph Theoretic Concepts in Computer Science (Proc. $W G$ '03), Lecture Notes Comput. Sci. Springer-Verlag, to appear.

57. P. Eades and S. Whitesides. The realization problem for Euclidean minimum spanning trees is NP-hard. Algorithmica, 16:60-82, 1996. (special issue on Graph Drawing, edited by G. Di Battista and R. Tamassia). 
58. B. Edler. Effiziente Algorithmen für flächenminimale Layouts von Bäumen. Diplomarbeit, Universität Passau, 1999.

59. H. ElGindy, D. Avis, and G. T. Toussaint. Applications of a two-dimensional hidden-line algorithm to other geometric problems. Computing, 31:191-202, 1983.

60. G. Even, S. Guha, and B. Schieber. Improved approximations of crossings in graph drawing and VLSI layout area. In Proceedings of the 32nd ACM Symposium on Theory of Computing (STOC'00), pages 296-305. ACM Press, 2000.

61. H. Everett. Visibility graph recognition. Report 231/90, Dept. Comput. Sci., Univ. Toronto, Toronto, ON, 1990. Ph.D. Thesis.

62. S. Felsner. Convex drawings of planar graphs and the order dimension of 3polytopes. Order, 18(1):19-37, 2001.

63. S. Felsner, G.Liotta, and S. Wismath. Straight line drawings on restricted integer grids in two and three dimensions. In P. Mutzel, M. Jünger, and S. Leipert, editors, Graph Drawing (Proc. GD '01), volume 2265 of Lecture Notes Comput. Sci., pages 328-342. Springer-Verlag, 2001.

64. Q.-W. Feng, R. F. Cohen, and P. Eades. Planarity for clustered graphs. In P. G. Spirakis, editor, Algorithms-ESA '95, Third Annual European Symposium, volume 979 of Lecture Notes in Computer Science, pages 213-226, Corfu, Greece, 25-27 Sept. 1995. Springer.

65. M. R. Garey and D. S. Johnson. Computers and Intractability: A Guide to the Theory of NP-Completeness. W. H. Freeman, New York, NY, 1979.

66. M. R. Garey, D. S. Johnson, F. P. Preparata, and R. E. Tarjan. Triangulating a simple polygon. Inform. Process. Lett., 7:175-179, 1978.

67. A. Garg. New results on drawing angle graphs. Comput. Geom. Theory Appl., 9(1-2):43-82, 1998.

68. A. Garg, M. T. Goodrich, and R. Tamassia. Planar upward tree drawings with optimal area. Internat. J. Comput. Geom. Appl., 6:333-356, 1996.

69. A. Garg and G. Liotta. Almost bend-optimal planar orthogonal drawings of biconnected degree-3 planar graphs in quadratic time. In J. Kratochvil, editor, Graph Drawing (Proc. GD '99), Lecture Notes Comput. Sci. Springer-Verlag, 1999.

70. A. Garg and A. Rusu. Straight-line drawings of binary trees with linear area and good aspect ratio. In M. Goodrich, editor, Graph Drawing (Proc. GD'02), volume 2528 of Lecture Notes Comput. Sci., pages 320-331. Springer-Verlag, 2002.

71. A. Garg and A. Rusu. Area-efficient drawings of outerplanar graphs. In Graph Drawing (Proc. GD 03), Lecture Notes in Comput. Sci., 2003. These proceedings.

72. A. Garg and R. Tamassia. A new minimum cost flow algorithm with applications to graph drawing. In S. C. North, editor, Graph Drawing (Proc. GD '96), Lecture Notes Comput. Sci. Springer-Verlag, 1997.

73. A. Garg and R. Tamassia. On the computational complexity of upward and rectilinear planarity testing. SIAM J. Comput., 31(2):601-625, 2001.

74. A. Garg, R. Tamassia, and P. Vocca. Drawing with colors. In Proc. 4th Annu. European Sympos. Algorithms, volume 1136 of Lecture Notes Comput. Sci., pages 12-26. Springer-Verlag, 1996.

75. S. K. Ghosh. On recognizing and characterizing visibility graphs of simple polygons. Discrete Comput. Geom., 17:143-162, 1997.

76. A. Gregori. Unit length embedding of binary trees on a square grid. Inform. Process. Lett., 31:167-172, 1989.

77. M. Grohe. Computing crossing numbers in quadratic time. In Proceedings of the 32nd ACM Symposium on Theory of Computing ( STOC'00), pages 231-236. ACM Press, 2000. 
78. C. Gutwenger, M. Jünger, S. Leipert, P. Mutzel, M. Percan, and R. Weiskircher. Advances in c-planarity testing of clustered graphs. In M. Goodrich and S. Kobourov, editors, Graph Drawing (Proc. GD'02), volume 2528 of Lecture Notes Comput. Sci., pages 220-325. Springer-Verlag, 2002.

79. C. Gutwenger and P. Mutzel. An experimental study of crossing minimization heuristics. In Graph Drawing (Proc. GD 03), Lecture Notes in Comput. Sci., 2003. These proceedings.

80. C. Gutwenger and P. Mutzel. Graph embedding with minimum depth and maximum external face. In Graph Drawing (Proc. GD 03), Lecture Notes in Comput. Sci., 2003. These proceedings.

81. C. Gutwenger, P. Mutzel, and R. Weiskircher. Inserting an edge into a planar graph. In Proc. Ninth Annual ACM-SIAM Symp. Discrete Algorithms (SODA '2001), pages 246-255, Washington, DC, 2001. ACM Press.

82. P. Healy and A. Kuusik. The vertex-exchange graph: a new concept for multi-level crossing minimization. In J. Kratochvíl, editor, Graph Drawing (Proc. GD '99), volume 1731 of Lecture Notes in Comput. Sci., pages 205-216. Springer-Verlag, 1999.

83. S. Hertel and K. Mehlhorn. Fast triangulation of the plane with respect to simple polygons. Inform. Control, 64:52-76, 1985.

84. J. W. Jaromczyk and G. T. Toussaint. Relative neighborhood graphs and their relatives. Proc. IEEE, 80(9):1502-1517, Sept. 1992.

85. M. Jünger, E. Lee, P. Mutzel, and T. Odenthal. A polyhedral approach to the multi-layer crossing number problem. In G. Di Battista, editor, Graph Drawing (Proc. GD '97), volume 1353 of Lecture Notes in Comput. Sci., pages 13-24. Springer-Verlag, 1997.

86. G. Kant. A new method for planar graph drawings on a grid. In Proc. 33rd Annu. IEEE Sympos. Found. Comput. Sci., pages 101-110, 1992.

87. G. Kant. Drawing planar graphs using the canonical ordering. Algorithmica, 16:4-32, 1996. (special issue on Graph Drawing, edited by G. Di Battista and R. Tamassia).

88. M. Kaufmann and R. Wiese. Embedding vertices at points: Few bends suffice for planar graphs. Journal of Graph Algorithms and Applications, 6(1):115-129, 2002.

89. M. Keil. Computing a subgraph of the minimum weight triangulation. Comput. Geom. Theory Appl., 4:13-26, 1994.

90. D. G. Kirkpatrick. A note on Delaunay and optimal triangulations. Inform. Process. Lett., 10(3):127-128, 1980.

91. G. W. Klau, K. Klein, and P. Mutzel. An experimental comparison of orthogonal compaction algorithms. In Graph Drawing (Proc. GD 2000), Lecture Notes Comput. Sci. Springer Verlag, 2001.

92. G. W. Klau and P. Mutzel. Optimal compaction of orthogonal grid drawings. In G. Cornuejols, R. E. Burkard, and G. J. Woeginger, editors, Integer Programming and Combinatorial Optimization, volume 1610 of Lecture Notes Comput. Sci., pages 304-319. Springer-Verlag, 1999.

93. F. T. Leighton. New lower bound techniques for VLSI. In Proc. 22nd Annu. IEEE Sympos. Found. Comput. Sci., pages 1-12, 1981.

94. F. T. Leighton and S. Rao. Multicommodity max-flow min-cut theorems and their use in designing approximation algorithms. Journal of the ACM, 46(6):787-832, 1999.

95. W. Lenhart and G. Liotta. Drawing outerplanar minimum weight triangulations. Inform. Process. Lett., 57(5):253-260, 1996. 
96. W. Lenhart and G. Liotta. Proximity drawings of outerplanar graphs. In Graph Drawing (Proc. GD '96), volume 1190 of Lecture Notes Comput. Sci. SpringerVerlag, 1997.

97. W. Lenhart and G. Liotta. The drawability problem for minimum weight triangulations. Theoret. Comp. Sci., 270:261-286, 2002.

98. C. Levcopoulos and D. Krznaric. Tight lower bounds for minimum weight triangulation heuristic. Inform. Process. Lett., 57:129-135, 1996.

99. C. Levcopoulos and D. Krznaric. A linear-time approximation schema for minimum weight triangulation of convex polygons. Algorithmica, 21:285-311, 1998.

100. A. Lingas. A new heuristic for minimum weight triangulation. SIAM J. Algebraic Discrete Methods, 8(4):646-658, 1987.

101. G. Liotta and G. Di Battista. Computing proximity drawings of trees in the 3dimensional space. In Proc. 4th Workshop Algorithms Data Struct., volume 955 of Lecture Notes Comput. Sci., pages 239-250. Springer-Verlag, 1995.

102. G. Liotta, A. Lubiw, H. Meijer, and S. H. Whitesides. The rectangle of influence drawability problem. Comput. Geom. Theory Appl., 10(1):1-22, 1998.

103. G. Liotta and H. Meijer. Voronoi drawings of trees. Comput. Geom. Theory Appl., 2003. to appear.

104. L. Lovasz, K. Vesztergombi, U. Wagner, and E. Welzl. Convex quadrilaterals and $k$-sets. Contemporary Mathematics, 2003.

105. A. Lubiw and N. Sleumer. Maximal outerplanar graphs are relative neighborhood graphs. In Proc. 5th Canad. Conf. Comput. Geom., pages 198-203, 1993.

106. S. Malitz and A. Papakostas. On the angular resolution of planar graphs. In Proc. 24th Annu. ACM Sympos. Theory Comput., pages 527-538, 1992.

107. G. K. Manacher and A. L. Zobrist. Neither the greedy nor the Delaunay triangulation of a planar point set approximates the optimal triangulation. Inform. Process. Lett., 9:31-34, 1979.

108. A. Mansfield. Determining the thickness of a graph is NP-hard. Math. Proc. Cambridge Philos. Soc., 93(9):9-23, 1983.

109. K. Mehlhorn, T. S. S. Näher, S. Schirra, M. Seel, R. Seidel, and C. Uhrig. Checking geometric programs or verification of geometric structures. Comput. Geom. Theory Appl., 12:85-113, 1999.

110. C. Monma and S. Suri. Transitions in geometric minimum spanning trees. Discrete Comput. Geom., 8:265-293, 1992.

111. P. Mutzel and R. Weiskircher. Computing optimal embeddings for planar graphs. In D.-Z. Du, P. Eades, V. Estivill-Castro, X. Lin, and A. Sharma, editors, Computing and Combinatorics, Proc. Sixth Annual Internat. Conf. (COCOON '2000), volume 1858 of Lecture Notes in Comput. Sci., pages 95-104. Springer-Verlag, 2000 .

112. J. Pach, F. Shahrokhi, and M. Szegedy. Applications of the crossing number. Algorithmica, 16:111-117, 1996. (special issue on Graph Drawing, edited by G. Di Battista and R. Tamassia).

113. J. Pach, T. Thiele, and G. Tóth. Three-dimensional grid drawings of graphs. In G. Di Battista, editor, Graph Drawing (Proc. GD '97), volume 1353 of Lecture Notes Comput. Sci., pages 47-51. Springer-Verlag, 1997.

114. J. Pach and G. Tóth. Graphs drawn with few crossings per edge. Combinatorica, 17:427-439, 1997.

115. M. Pizzonia and R. Tamassia. Minimum depth graph embedding. In Proc. European Symposium on Algorithms (ESA 2000), Lecture Notes in Computer Science. Springer-Verlag, 2000. 
116. F. P. Preparata and M. I. Shamos. Computational Geometry: An Introduction. Springer-Verlag, 3rd edition, Oct. 1990.

117. S. Rahman, N. Egi, and T. Nishizeki. No-bend orthogonal drawings of subdivisions of planar triconnected cubic graphs. In Graph Drawing (Proc. GD 03), Lecture Notes in Comput. Sci., 2003. These proceedings.

118. D. Rappaport and H. Meijer. Computing the minimum weight triangulation of a set of linearly ordered points. Information Processing Letters, 42:35-38, 1992.

119. R. Richter and C. Thomassen. Relations between crossing numbers of complete and complete bipartite graphs, February 1997.

120. P. Rosenstiehl and R. E. Tarjan. Rectilinear planar layouts and bipolar orientations of planar graphs. Discrete Comput. Geom., 1(4):343-353, 1986.

121. E. R. Scheinerman and H. S. Wilf. The rectilinear crossing number of a complete graph and Sylvester's "four point problem" of geometric probability. Amer. Math. Monthly, 101(10):939-943, 1994.

122. W. Schnyder. Planar graphs and poset dimension. Order, 5:323-343, 1989.

123. W. Schnyder. Embedding planar graphs on the grid. In Proc. 1st ACM-SIAM Sympos. Discrete Algorithms, pages 138-148, 1990.

124. W. Schnyder and W. T. Trotter. Convex embeddings of 3-connected plane graphs. Abstracts of the AMS, 13(5):502, 1992.

125. C.-S. Shin, S. K. Kim, and K.-Y. Chwa. Area-efficient algorithms for straight-line tree drawings. Comput. Geom. Theory Appl., 15:175-202, 2000.

126. K. Sugiyama, S. Tagawa, and M. Toda. Methods for visual understanding of hierarchical systems. IEEE Trans. Syst. Man Cybern., SMC-11(2):109-125, 1981.

127. O. Sýkora and I. Vrťo. On VLSI layouts of the star graph and related networks. The VLSI Journal, 17:83-93, 1994.

128. R. Tamassia. On embedding a graph in the grid with the minimum number of bends. SIAM J. Comput., 16(3):421-444, 1987.

129. G. Toussaint. Efficient triangulation of simple polygons. Visual Comput., 7:280295, 1991.

130. G. Vijayan and A. Wigderson. Rectilinear graphs and their embeddings. SIAM J. Comput., 14:355-372, 1985.

131. V. Vijayan. Geometry of planar graphs with angles. In Proc. 2nd Annu. ACM Sympos. Comput. Geom., pages 116-124, 1986.

132. V. Waddle and A. Malhotra. An E log E line crossing algorithm for levelled graphs. In J. Kratochvil, editor, Graph Drawing (Proc. GD '99), Lecture Notes Comput. Sci., pages 59-70. Springer-Verlag, 1999.

133. C. A. Wang, F. Y. Chin, and B. Yang. Triangulations without minimum weight drawing. In Algorithms and Complexity (Proc. CIAC 2000), volume 1767 of Lecture Notes Comput. Sci., pages 163-173. Springer-Verlag, 2000.

134. T. C. Woo and S. Y. Shin. A linear time algorithm for triangulating a point-visible polygon. ACM Trans. Graph., 4(1):60-70, 1985.

135. D. R. Wood. Queue layouts, tree-width, and three-dimensional graph drawing. In 22nd Foundations of Software Technology and Theoretical Comput. Sci. (FSTTCS '02), volume 2556 of Lecture Notes in Comput. Sci., pages 348-359, 2002.

136. H. Zhang and X. He. Compact visibility representations and straight-line grid embedding of plane graphs. In Proc. 8th International Workshop on Algorithms and Data Structures (WADS '03), volume 2748 of LNCS, pages 493-504. Springer, 2003. 